\title{
A Network Pharmacology Approach to Uncover the Pharmacological Mechanism of XuanHuSuo Powder on Osteoarthritis
}

\author{
Huaqi Tang, Shuaibing He, Xinyue Zhang, Shilin Luo, Baixia Zhang, Xiaojie Duan, \\ Zhiqian Zhang, Wenqi Wang, Yun Wang, and Yikun Sun
}

School of Chinese Materia Medica, Beijing University of Chinese Medicine, Beijing 100102, China

Correspondence should be addressed to Yun Wang; wangyun@bucm.edu.com and Yikun Sun; sunyik@163.com

Received 9 December 2015; Accepted 3 March 2016

Academic Editor: Lixing Lao

Copyright (C) 2016 Huaqi Tang et al. This is an open access article distributed under the Creative Commons Attribution License, which permits unrestricted use, distribution, and reproduction in any medium, provided the original work is properly cited.

\begin{abstract}
As the most familiar type of arthritis and a chronic illness of the joints, Osteoarthritis (OA) affects a great number of people on the global scale. XuanHuSuo powder (XHSP), a conventional herbal formula from China, has been extensively applied in OA treatment. Nonetheless, its pharmacological mechanism has not been completely expounded. In this research, a network pharmacology approach has been chosen to study the pharmacological mechanism of XHSP on OA, and the pharmacology networks were established based on the relationship between four herbs found in XHSP, compound targets, and OA targets. The pathway enrichment analysis revealed that the significant bioprocess networks of XHSP on OA were regulation of inflammation, interleukin-1 $\beta$ (IL-1 $\beta$ ) production and nitric oxide (NO) biosynthetic process, response to cytokine or estrogen stimuli, and antiapoptosis. These effects have not been reported previously. The comprehensive network pharmacology approach developed by our research has revealed, for the first time, a connection between four herbs found in XHSP, corresponding compound targets, and OA pathway systems that are conducive to expanding the clinical application of XHSP. The proposed network pharmacology approach could be a promising complementary method by which researchers might better evaluate multitarget or multicomponent drugs on a systematic level.
\end{abstract}

\section{Introduction}

Osteoarthritis (OA) refers to an illness of the joints and bones featured by progressive and chronic structural damage [1], particularly the destruction of articular cartilage, with joint instability, chronic pain, and stiffness as clinical symptoms [2]. Gender, age, obesity, traumatic injury, and metabolic dysfunction can be considered as the main OA risk factors [3]. Currently, most OA treatment strategies focus on reducing symptoms, enhancing function, and delaying time to surgery. There are three types of therapeutic agents, nonsteroidal antiinflammatory drugs (NSAIDs), disease-modifying OA drugs (DMOADs), and biological and steroid response modifiers, which can relieve severity, reduce progression of disease, and avoid subsequent damage to the joints [4]. Although these strategies show excellent effects on OA, reports show that long-term application will cause side effects on the gastrointestinal tract [5].
As an important aspect of the complementary and alternative medical system, traditional Chinese medicine (TCM) has been widely utilized in the treating of OA for centuries [6] and has been proven efficient in relieving OA severity. In terms of the concept of TCM, OA can be classified as producing "arthromyodynia" (blockage syndrome, Bi syndrome, or Bi Zheng) [7]. However, application of TCM has been blocked by the absence of scientific comprehension regarding its mechanism. Therefore, it is important to explore and reveal the TCM mechanism.

The use of XuanHuSuo powder (XHSP) was recorded in $\mathrm{Pu} J i$ Fang, which is regarded as the greatest herbal formula book in China and was written by Su Zhu in 1406 during the early Ming Dynasty. This formula is prepared from four Chinese herbs including Angelica sinensis (Oliv.) Diels (Dang Gui), Corydalis yanhusuo W. T. Wang (Yan Hu Suo), Psoralea corylifolia L. (Bu Gu Zhi), and Achyranthes bidentata $\mathrm{Bl}$. 
(Niu Xi). Based on TCM theory, multiple agents in one formula should operate cooperatively. In terms of XHSP, Angelica sinensis and Corydalis yanhusuo are important for activating blood and strengthening analgesic functions; Achyranthes bidentata and Psoralea corylifolia can strengthen bone and tonify kidney; Achyranthes bidentata can emphasize actions of the formula on a particular region of the patient's body that suffers from OA or combine the action of other herbs in the formula. Recent studies show that compounds in each herb have curative efficacy for OA [8-12]; thus, the XHSP formula might be a novel therapeutic strategy for OA. However, the pharmacological mechanism has not been completely clarified.

Chinese herbal formulas (Fu-Fang) are multitarget and multicomponent agents that realize their particular therapeutic efficacy through regulation of the molecular network of body systems utilizing its active components [13]. Thus, new approaches and tactics are required to achieve systematic and comprehensive understanding of the herbal formula's mechanism. Hopkins has proposed the concept of network pharmacology [14] to investigate the influence or intervention of drugs and to show the synergism law of multicomponent drugs to seek high efficacy and low toxicity of multiple target medications. Meanwhile, the herbal formula is regarded as a multitarget and multicomponent therapeutic that meets the requirement of curing complicated illnesses in an integrated manner; thus, the methodology of network pharmacology can be used to pursue transcendental knowledge regarding the rules of combination in the formulas [15]. Therefore, a comprehensive network pharmacology method has been selected to understand the pharmacological mechanism of XHSP on OA, which offers a valuable opportunity for indepth comprehension of the mechanism for inversing this illness-associated imbalanced network.

\section{Materials and Methods}

\subsection{Data Preparation}

2.1.1. Composite Compounds of Each Herb in XHSP. Four hundred and thirty-five compounds from the four herbs found in XHSP were retrieved from references, TCM Database@Taiwan [16] (http://tcm.cmu.edu.tw/, updated in July 2013), which is the most comprehensive TCM database in the world, and the Traditional Chinese Medicine Systems Pharmacology Database [17] (TcmSP ${ }^{\mathrm{TM}}$, http://lsp.nwsuaf .edu.cn/, updated on May 31, 2014), a unique system pharmacology platform designed for Chinese herbal medicines. Thirty-two compounds in Psoralea corylifolia, 88 in Corydalis yanhusuo, 143 in Angelica sinensis, and 172 in Achyranthes bidentata were collected. The details are described in Table S1 (see Supplementary Material available online at http://dx.doi.org/10.1155/2016/3246946).

2.1.2. Compound Targets for Each Herb in XHSP. All of the compound targets of each herb found in XHSP were collected from Stitch [18] (http://stitch.embl.de/, ver. 4.0) with the species limited as "Homo sapiens" and a confidence score
$>0.4$. The compounds without relevant information were removed. Stitch is a resource to explore interactions between chemicals and proteins. Thus, we collected distinct targets related to the compounds in each herb found in XHSP. The details are described in Table S2.

2.1.3. OA Targets. The OA targets were gathered from the therapeutic target database [19] (http:/bidd.nus.edu.sg/ group/ttd/ttd.asp, updated on September 15, 2013), which offers information about nucleic acid targets and therapeutic proteins. A total of $24 \mathrm{OA}$ targets were gathered. The details are described in Table S3.

2.1.4. Protein-Protein Interaction Data. Protein-protein interaction (PPI) data were a term from String [20] (http://string-db.org/, ver. 10), with the species limited to "Homo sapiens" and a confidence score $>0.4$, Database of Interacting Proteins [21] (DIP ${ }^{\mathrm{TM}}$, http://dip.doe-mbi.ucla.edu/ dip/Main.cgi, updated on February 4, 2014), InAct [22] (http://www.ebi.ac.uk/intact/, ver. 4.2.1), and Molecular INTeraction database [23] (MINT, http://mint.bio.uniroma2 .it/mint/Welcome.do, updated on August 30, 2011). Among them, String is a database of known and forecasted proteinprotein interactions. DIP catalogs experimentally determined interactions between proteins and combines information from a variety of sources to create a single or consistent set of protein-protein interactions. InAct provides an open source database and analysis tools for molecular interaction data. And MINT focuses on experimentally verified protein-protein interactions mined from literature.

\subsection{Network Construction}

2.2.1. Network Construction Method. Network construction was performed as follows: (1) a compound-compound target network was established by linking chemical compounds and corresponding targets [24]; (2) herb-compound targetOA target network was built by connecting the four XHSP herbs, corresponding compound targets, and OA targets that interacted with compound targets; and (3) compound target-OA target-other human proteins' PPI network was constructed by connecting compound targets, OA targets, and other human proteins that are directly interacting with the two targets.

All of the networks could be generated by utilizing the network visualization software Cytoscape [25] (http://cytoscape.org/, ver. 3.2.1), which is used to visualize biological pathways and networks of molecular interactions and to interact with these networks via profiles of gene expression, annotations, and other state data. The software then offers a basic set of features for data integration, analysis, and visualization for complicated network analysis.

2.2.2. Network Topological Feature Set Definition. For every node in the network, three indices were applied to assess its topological features. "Degree" stands for the number of links or edges between a node and other nodes in a network [26]. "Node betweenness" evaluates the participation of a node in 


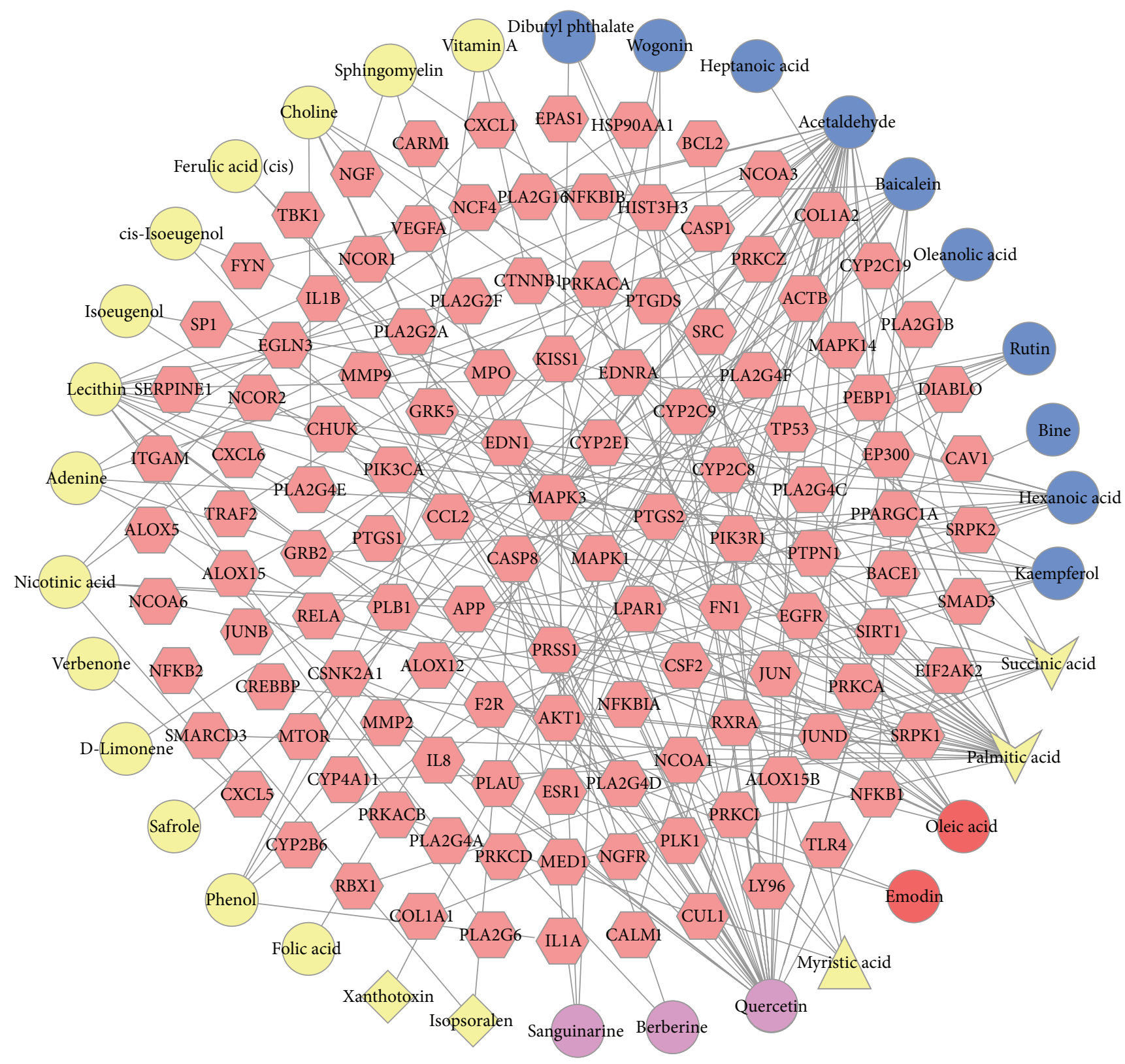

FIgURE 1: A compound-compound target network for XHSP on treating OA was built by linking 34 compounds and 122 compound targets. (Red hexagons represent compound targets. Yellow circles, blue circles, red circles, yellow diamonds, purple circles, yellow vee, and yellow triangle stand for the compounds contained in Angelica sinensis, Achyranthes bidentata, Corydalis yanhusuo, Psoralea corylifolia, Corydalis yanhusuo and Achyranthes bidentata, Angelica sinensis and Achyranthes bidentata, and Psoralea corylifolia and Angelica sinensis and Achyranthes bidentata, resp.)

the shortest parts of a network and reflects the capability of nodes to manage the rate of information flow in the network [27]. "Closeness" is the inverse of the sum of the distance from node $i$ to other nodes, which is a measurement of how long it will take to spread information from node $i$ to other nodes [28]. The higher the degree, node betweenness, and closeness of a node are, the more significant it is in the network.

2.3. Pathway Enrichment Analysis. The Database for Annotation, Visualization and Integrated Discovery [29] (DAVID, https://david.ncifcrf.gov/home.jsp, ver. 6.7) was applied for Gene Ontology (GO) enrichment analysis.

\section{Results and Discussion}

3.1. Compound-Compound Target Network Analysis. The compound-compound target network is shown in Figure 1, including 156 nodes (34 compounds in XHSP yielded 122 compound targets) and 220 edges. In this network, targets in the interior circle show more interactions with compounds 


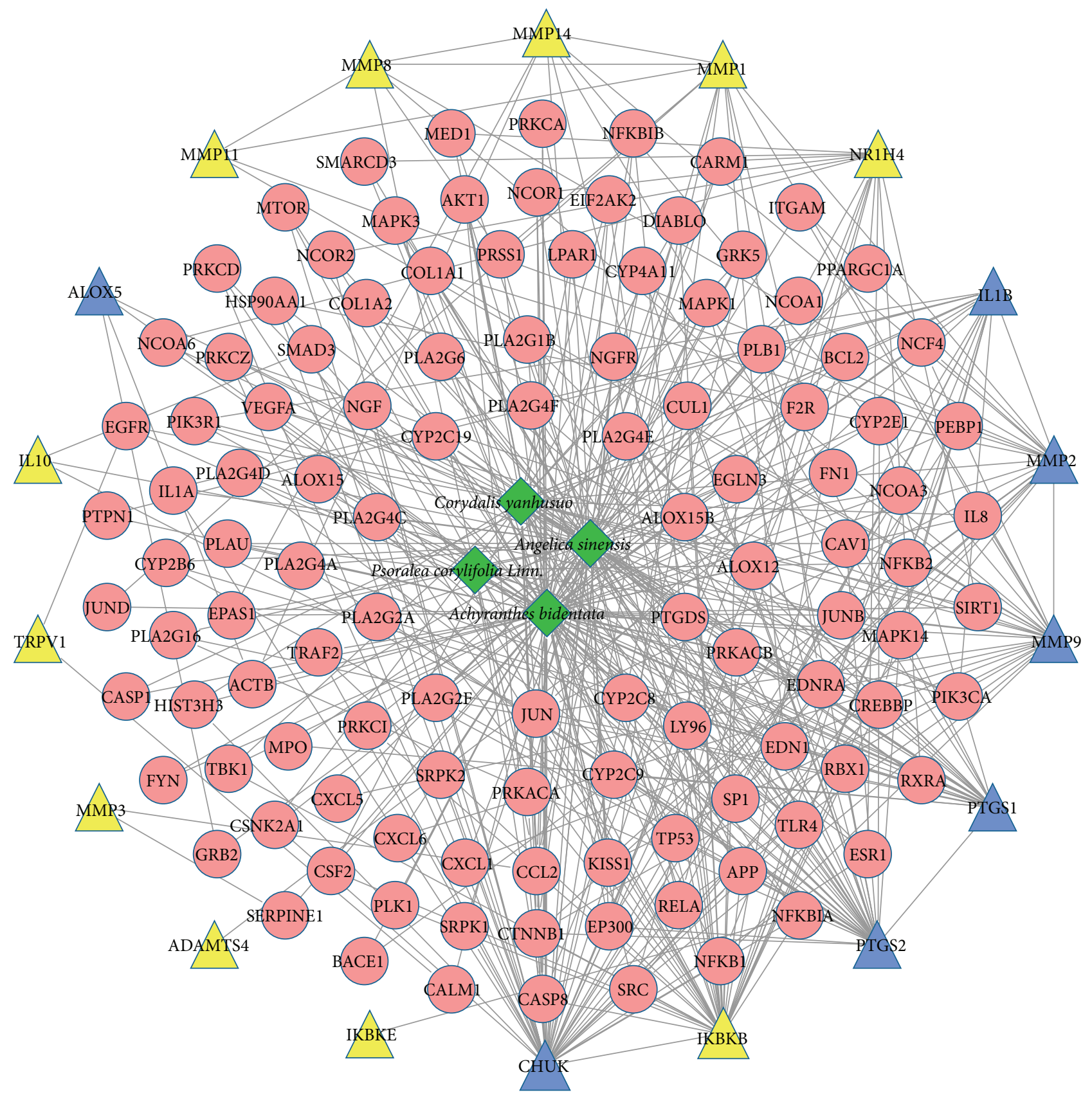

FIGURE 2: Herb-compound target-OA target network. (Green diamonds, red circles, yellow triangles, and blue triangles represent herbs, compound targets, OA targets, and compound targets/OA targets, resp.)

than those in the exterior. This indicates that a great number of compound targets can be regulated by multiple compounds instead of just one. For instance, EDN1 and PTGS2 are regulated by multiple ingredients including palmitic acid and quercetin. Besides, MAPK3, MAPK1, and CCL2 can also be regulated by more than one. Therefore, we could obtain an approximate observation of the relationship between compounds and compound targets from this network.

3.2. Herb-Compound Target-OA Target Network Analysis. The herb-compound target-OA target network was constructed in order to identify the relationship between the four herbs in XHSP and the corresponding compound targets and OA targets. In Figure 2, the network was composed of 137 nodes (four herbs, 115 compound targets, 11 OA targets, and seven compound targets/OA targets) and 458 edges. Achyranthes bidentata showed the highest degree of distribution followed by Angelica sinensis and Corydalis yanhusuo, the links of which with other nodes were all over 40 , thus, leading to their significance in the network.

In Figure 3, according to the enrichment analysis, compound targets and OA targets were significantly associated with the regulation of nitric oxide (NO) biosynthetic process (GO ID: 45428, fold enrichment $=26.37, P<0.001$ ), response 


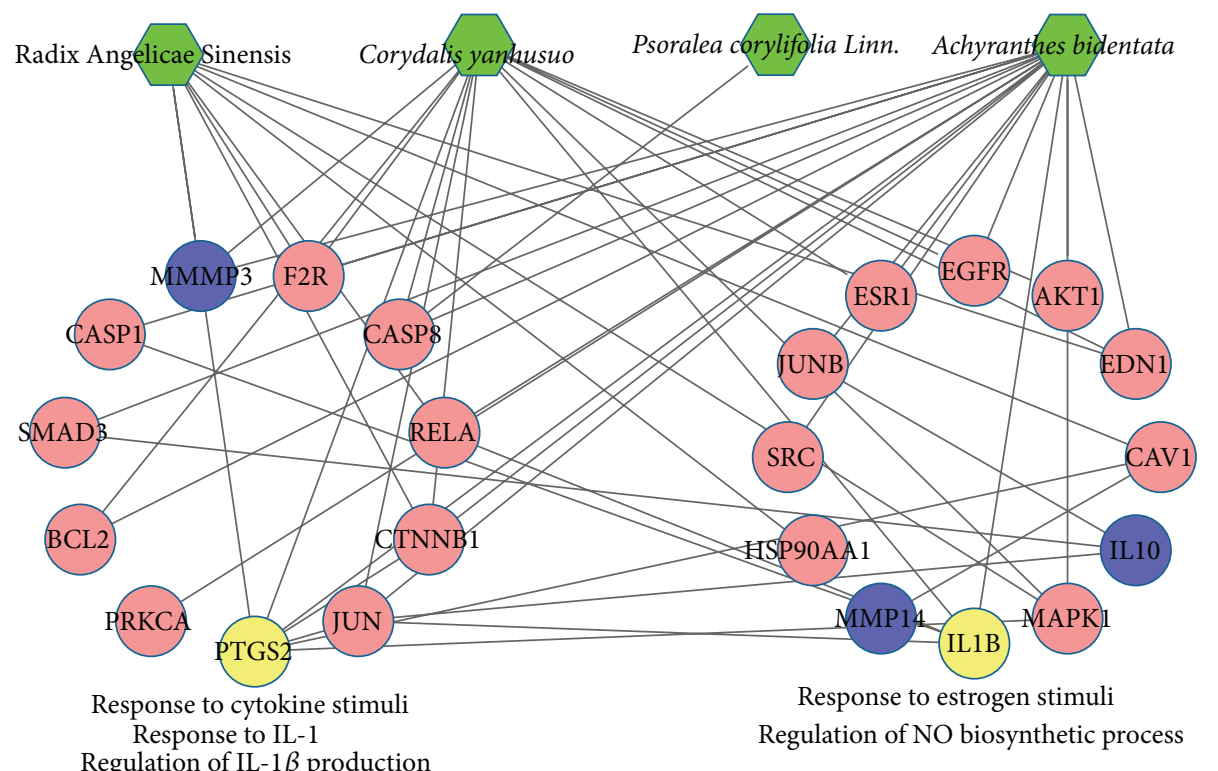

FIGURE 3: According to the associated biological processes or pathways, compound targets of XHSP and OA targets were related to various molecular mechanisms of OA. (Green nodes, pink circles, blue circles, and yellow circles represent four herbs in XHSP, compounds targets, OA targets, and compound targets/OA targets, resp.)

to interleukin-1 (IL-1) (GO ID: 70555, fold enrichment = 17.95, $P=0.012)$, regulation of interleukin-1 $\beta(\mathrm{IL}-1 \beta)$ production (GO ID: 32651, fold enrichment $=15.26, P=0.016$ ), response to cytokine stimuli (GO ID: 34097 , fold enrichment $=12.88$, $P<0.001$ ), and response to estrogen stimuli (GO ID: 43627, fold enrichment $=8.72, P<0.001$ ). The details are described in Table S4.

The latest medical knowledge shows that the immune system is a key element in the progression of OA [30], and immune cytokines play a crucial role [31] by disturbing the anabolism and catabolism processes of tissue cells in joints [32]. The IL-1 family, which includes IL- $1 \alpha$, IL-1 $\beta$, IL1 receptor antagonist (IL-1Ra), and IL-18 [33], is significant in strengthening inflammatory reactions [34] and accelerating destruction of cartilage tissue [35]. This family of cytokines is especially involved in enhancing matrix metalloproteinases (MMPs) expression, which can speed up the synthesis and release of MMP zymogen, particularly MMPs 2, 3, 9, and 13, which increase matrix molecule degradation. IL-1 can also promote chondrocytes and synoviocytes to cause synovitis by producing prostaglandin E2 (PGE2) [36], which can reinforce the cartilage degradation effect of IL-1 and then accelerate OA progress. The increasing expression of IL1 in OA synoviocytes can intensify the synovitis and the production of inflammatory cytokines and MMPs [37]. This is further confirmed by the existence of IL-1 in OA synovitis and degradation of the cartilage matrix [38].

According to reports, high levels of IL- $1 \beta$ can be found in the synovial membrane, synovial fluid, cartilage tissue, and subchondral bone tissue of OA patients [39]. This leads to a decrease in the synthesis of major extracellular matrix (ECM) components by inhibiting anabolic activity and increasing proteolytic enzyme yield in chondrocytes and subsequently degrading articular cartilage [40]. It also indicates that the degradation of cartilage in mice, rabbits, dogs, and pigs might be reduced by blocking IL-1 $\beta$ activity [41].

A number of proinflammatory factors, such as IL-1 $\beta, \mathrm{NO}$, and their downstream mediators, can activate the expression of MMPs [42], which particularly decrease the proteoglycans and native collagens [43]. For instance, NO is produced by inducible nitric oxide synthase (iNOS) [44]. Increasing levels of NO and iNOS could inhibit collagen and proteoglycan biosynthesis in OA patients [45], leading to the activation of MMPs and the inflammatory response [46]. Thus, NO shows the function of catabolism in OA cartilage.

Estrogen is closely associated with knee Osteoarthritis in middle-aged females [47]. According to recent research, females are more vulnerable to severe keen OA than males, especially after menopause [48]. Furthermore, the symptoms are higher in women than in men with the same level of radiographic damage. Thus, it could be hypothesized that the onset or progression of knee OA might relate to estrogen [49]. At the same time, other research found that estrogen deficiency may affect periarticular muscles, synovial lining, ligaments, articular capsule, and subchondral bone during the OA process [50]. All of this research indicates a potential protective role of estrogens in the OA process [51].

\subsection{Compound Target-OA Target-Other Human Proteins' PPI} Network Analysis. To evaluate the significance of compound targets, a compound target-OA target-other human proteins' PPI network was constructed with 609 nodes (115 compound targets, 12 OA targets, seven compound targets/OA targets, and 475 other human proteins interacting with compound 


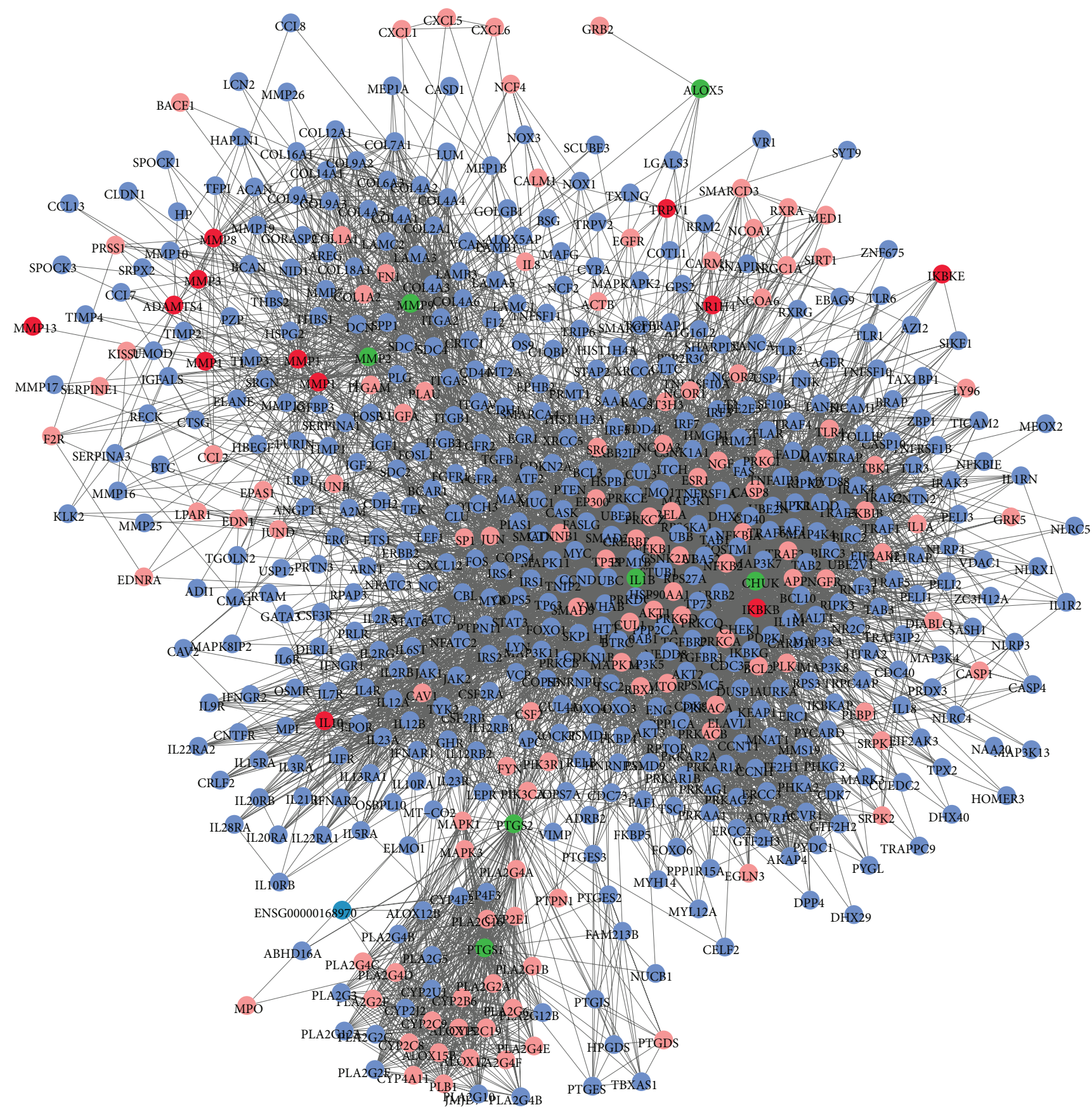

FIgURE 4: Compound target-OA target-other human proteins' PPI network. (Pink nodes, red nodes, green nodes, and blue nodes represent compound targets, OA targets, compound targets/OA targets, and other human proteins interacting with compound targets or OA targets, resp.)

targets or OA targets) and 7379 edges (Figure 4). Three topological features of each node in the network were calculated to find the major nodes. Thus, 72 nodes with an average value of degree $\geq 24.19$, node betweenness $\geq 0.00244$, and closeness $\geq 0.4097$ could be considered as major nodes (the details are described in Table S5). Finally, 41 major nodes, which intersected with compound targets, could be recognized as potential targets of XHSP.

Furthermore, the direct interaction network between the 72 major nodes was established with 41 nodes (16 compound targets, four compound targets and/or OA targets, and 21 other human proteins interacting with compound targets or OA targets) and 318 edges. As shown in Figure 5, the major nodes could be categorized into various functional modules, including regulation of NO biosynthetic process (GO ID: 45428 , fold enrichment $=28.23, P<0.001$ ), response to estrogen stimuli (GO ID: 43627, fold enrichment $=18.15, P<$ 0.001 ), antiapoptosis (GO ID: 6916, fold enrichment $=12.95$, $P<0.001$ ), regulation of inflammatory response (GO ID: 50727, fold enrichment $=10.03, P=0.007$ ), and apoptosis 


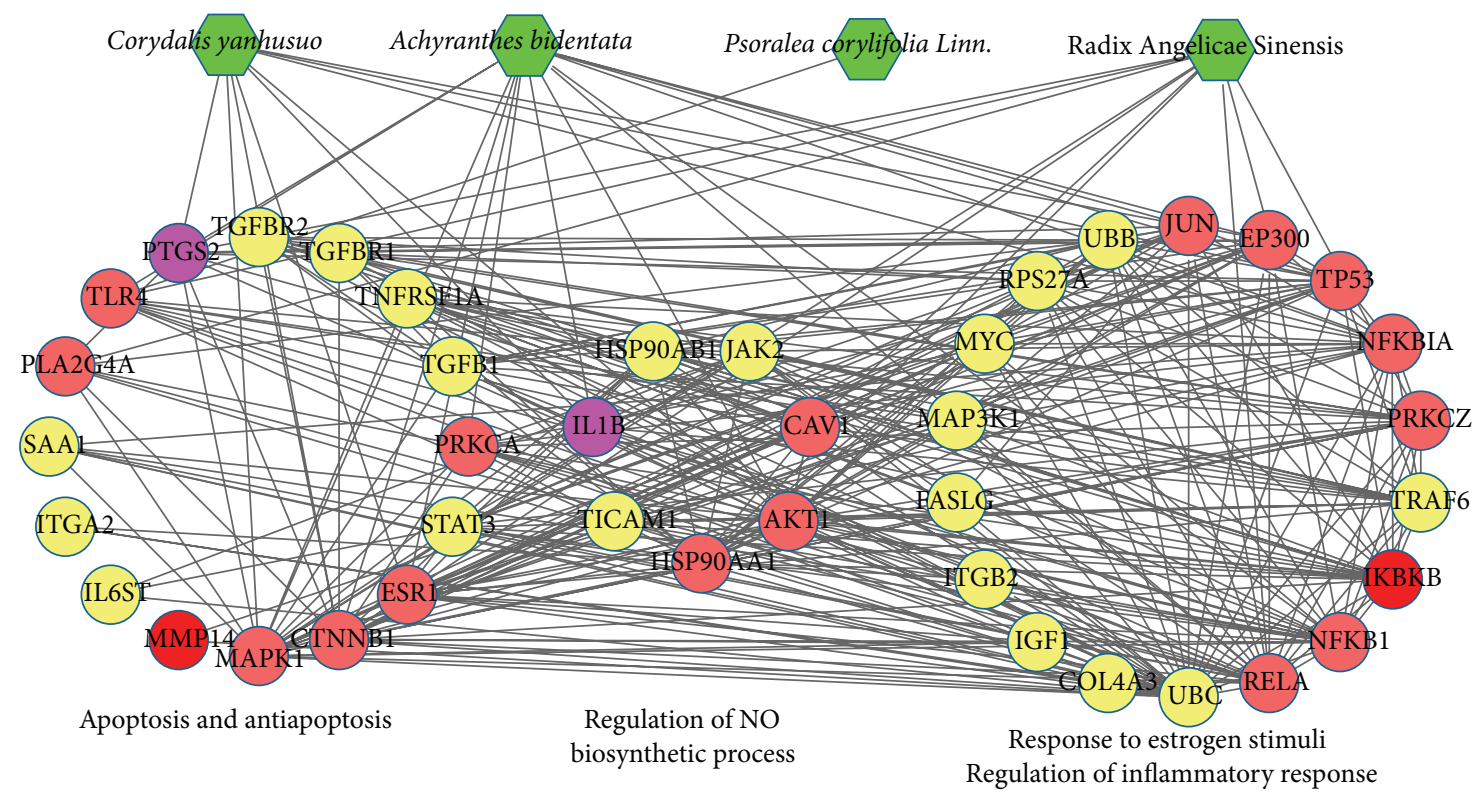

FIGURE 5: Direct interaction network between 41 major nodes in the XHSP compound target-OA target-other human proteins' PPI network. According to the correlative pathways, those nodes were divided into different functional modules, which were all related to OA. (Green diamonds, pink circles, red circles, purple circles, and yellow circles represent herbs in XHSP, compound targets, OA targets, compound targets/OA targets, and other human proteins interacting with compound targets or OA targets, resp.)

(GO ID: 6915 , fold enrichment $=5.70, P<0.001$ ). The details are described in Table S6.

Research has shown that cartilage is sensitive to estrogen [52], a type of antiresorptive agent that can attenuate osteoclastic resorption and osteoclastogenesis [53]. Meanwhile, the beneficial effect of estrogen on OA prevention has been confirmed by animal experiment with estrogen replacement therapy (ERT) treatment [54]. In that study, fewer cartilage lesions were detected in ERT group than control group. In addition, experiments in rats [55], female rabbits [56], and monkeys [57] show that estrogen deprivation can increase bone turnover [58], accelerate cartilage breakdown [59], and increase bone resorption resulting in worsening of $\mathrm{OA}$.

$\mathrm{OA}$ is characterized by inhibition of anabolic factor production and release of more catabolic factors [60], which leads to greater cartilage damage and a more severe inflammatory response [61]. Inflammatory cytokines play a crucial role [62] in disturbing the anabolism and catabolism of the joints [63]. Among these inflammatory cytokines, IL- $1 \beta$ is regarded as one of the most significant cytokines in OA pathogenesis [64]. IL-1 $\beta$ increases the expression of MMPs, which are crucial in the degradation of cartilage in OA [65], and induces the production of inflammatory mediators, which can result in clinical presentation of OA.

The treatment of OA is mainly based on the application of NSAIDs [66]; however, long-term application results in side effects on the gastrointestinal tract. In this regard, our data show that OA can be weakened by XHSP through reversing estrogen depletion and regulating inflammatory response by reducing the generation of inflammatory cytokines.
As two risk factors for $\mathrm{OA}$, mechanical stress and inflammation are both related to NO upregulation [67]. NO is detrimental to the joint and makes contributions to the pathogenesis of OA [68] by modulating cytokine expression, suppressing matrix collagen and proteoglycan synthesis, activating MMPs, suppressing proliferation of chondrocytes, and promoting chondrocyte apoptosis [69]. NO manufactured by iNOS plays a complicated role in OA pathology and pain [70]. Utilizing iNOS or NO synthase inhibitors has resulted in great interest in therapeutic interventions, such as $\mathrm{N}^{6}$-(1-iminoethyl)-L-lysine hydrochloride [69] and Smethylisothiourea [71], which are effective in restraining OA progression. Therefore, the role of XHSP in the adjustment process of NO biosynthesis during OA should be an emphasis of further research.

Apoptosis, especially chondrocyte apoptosis, has been shown to have a close relationship with severity and progression of OA [72]. Chondrocytes are crucial in anaboliccatabolic balance for tissue function and matrix maintenance [73] and are capable of responding to structural changes in cartilage matrix [74]. Therefore, inhibition of chondrocyte apoptosis and maintenance of chondrocytes in a healthy condition might be a potential strategy against cartilage degeneration progression [75], preserving the complete cartilage and preventing its degeneration [76]. Current research has shown that berberine chloride is advantageous to matrix synthesis and cell survival in IL- $1 \beta$ stimulated chondrocytes and shows great therapeutic potential for cartilage repair in rat OA models [77]. Our study shows that XHSP directly interacts with chondrocyte apoptosis. Therefore, it can be 
hypothesized that XHSP acts via antiapoptosis activities to offer a protective effect on OA chondrocytes.

\section{Conclusion}

Perfect therapeutic strategies for OA have not been discovered yet, except for conventional strategies such as analgesics and NSAIDs. However, the side effects of long-term application of these treatments lead to increasing usage of TCM, which treats illnesses with various formulas to fit the patients' symptoms and constitutions. The TCM herbal formula is efficient in treating complicated illnesses such as OA due to its multicomponent and multitarget agent property, which could generate higher degrees of effectiveness with less toxic side effects. Unlike western medicine, TCM treats illness in a more systematic and holistic way. However, many studies are still applying the traditional research idea, "one-drugone-target-one-illness," which ignores the multitarget and multicomponent characteristic of TCM formula.

Due to the rapid development of bioinformatics, the network approach has become an innovative and effective means to uncover the molecular mechanisms underlying the curative effect of TCM formulas from a systematic viewpoint. In this research, we combined a number of network-based computational methods and algorithm-based approaches to predict targets, construct networks, and illuminate the molecular synergy of XHSP for OA. This provides clues to study the synergetic effects of each herb and pinpoint the major targets of XHSP and the corresponding molecular pathways on OA.

According to the prediction of network pharmacology, several signal pathways of XHSP acting on OA have been found for the first time. Regulation of inflammation, IL-1 $\beta$ production, NO biosynthetic processes, response to cytokines or estrogen stimuli, and antiapoptosis pathways were all identified as signaling pathways for the actions of XHSP on OA. All of these results are expected to help identify novel curative efficacy and take full clinical advantage of XHSP. In general, these results and network pharmacology approach could not only contribute to uncovering of the prescription rules of Chinese herb formula in clinical application but also demonstrate a holistic version of herbal remedies and facilitate the pharmacological evaluation of herb treatments. Based on our work, we believe that evaluating the efficacy of TCM formulas and uncovering the pharmacological mechanism on a systematic level will be a promising complementary and alternative method for future studies.

\section{Competing Interests}

The authors declare that they have no competing interests.

\section{Authors' Contributions}

Huaqi Tang and Shuaibing He contributed equally to this work.

\section{Acknowledgments}

This work is supported by the National Natural Science Foundation of China (Grant nos. 81173521 and 81373942).

\section{References}

[1] Y. Xu, Q. Liu, Z.-L. Liu, L. Lim, W.-H. Chen, and N. Lin, "Treatment with SiMiaoFang, an anti-arthritis Chinese herbal formula, inhibits cartilage matrix degradation in osteoarthritis rat model," Rejuvenation Research, vol. 16, no. 5, pp. 364-376, 2013.

[2] J. Shen and D. Chen, "Recent progress in osteoarthritis research," Journal of the American Academy of Orthopaedic Surgeons, vol. 22, no. 7, pp. 467-468, 2014.

[3] D. J. Hunter, D. Schofield, and E. Callander, "The individual and socioeconomic impact of osteoarthritis," Nature Reviews Rheumatology, vol. 10, no. 7, pp. 437-441, 2014.

[4] L. Brosseau, G. A. Wells, G. P. Kenny et al., "The implementation of a community-based aerobic walking program for mild to moderate knee osteoarthritis (OA): a knowledge translation (KT) randomized controlled trial (RCT): part I: the Uptake of the Ottawa Panel clinical practice guidelines (CPGs)," BMC Public Health, vol. 12, article 871, 2012.

[5] J.-Y. Reginster, A. Neuprez, M.-P. Lecart, N. Sarlet, and O. Bruyere, "Role of glucosamine in the treatment for osteoarthritis," Rheumatology International, vol. 32, no. 10, pp. 2959-2967, 2012.

[6] F.-P. Chen, C.-M. Chang, S.-J. Hwang, Y.-C. Chen, and F.J. Chen, "Chinese herbal prescriptions for osteoarthritis in Taiwan: analysis of National Health Insurance dataset," BMC Complementary and Alternative Medicine, vol. 14, article 91, 2014.

[7] H. Yan, Y. Su, L. Chen et al., "Rehabilitation for the management of knee osteoarthritis using comprehensive traditional Chinese medicine in community health centers: study protocol for a randomized controlled trial," Trials, vol. 14, article 367, 2013.

[8] L. Yang, X. Sun, and X. Geng, "Effects of psoralen on chondrocyte degeneration in lumbar intervertebral disc of rats," Pakistan Journal of Pharmaceutical Sciences, vol. 28, supplement 2, pp. S667-S670, 2015.

[9] K. Xu, X. Pan, Y. Sun, W. Xu, L. Njunge, and L. Yang, "Psoralen activates cartilaginous cellular functions of rat chondrocytes in vitro," Pharmaceutical Biology, vol. 53, no. 7, pp. 1010-1015, 2015.

[10] M. Kubo, H. Matsuda, K. Tokuoka, S. Ma, and H. Shiomoto, "Anti-inflammatory activities of methanolic extract and alkaloidal components from Corydalis tuber," Biological \& Pharmaceutical Bulletin, vol. 17, no. 2, pp. 262-265, 1994.

[11] J. Magdalou, L.-B. Chen, H. Wang et al., "Angelica sinensis and osteoarthritis: a natural therapeutic link?" Bio-Medical Materials and Engineering, vol. 25, supplement 1, pp. S179-S186, 2015.

[12] X. Weng, P. Lin, F. Liu et al., "Achyranthes bidentata polysaccharides activate the $\mathrm{Wnt} / \beta$-catenin signaling pathway to promote chondrocyte proliferation," International Journal of Molecular Medicine, vol. 34, no. 4, pp. 1045-1050, 2014.

[13] Y. Zhang, X. Guo, D. Wang et al., "A systems biology-based investigation into the therapeutic effects of Gansui Banxia Tang on reversing the imbalanced network of hepatocellular carcinoma," Scientific Reports, vol. 4, Article ID 04154, 10 pages, 2014. 
[14] A. L. Hopkins, "Network pharmacology: the next paradigm in drug discovery," Nature Chemical Biology, vol. 4, no. 11, pp. 682690, 2008.

[15] Q. Guo, M. Zhong, H. Xu, X. Mao, Y. Zhang, and N. Lin, "A systems biology perspective on the molecular mechanisms underlying the therapeutic effects of Buyang Huanwu decoction on ischemic stroke," Rejuvenation Research, vol. 18, no. 4, pp. 313-325, 2015.

[16] C. Y.-C. Chen, “TCM Database@Taiwan: the world's largest traditional Chinese medicine database for drug screening in silico," PLoS ONE, vol. 6, no. 1, Article ID e15939, 2011.

[17] J. Ru, P. Li, J. Wang et al., "TCMSP: a database of systems pharmacology for drug discovery from herbal medicines," Journal of Cheminformatics, vol. 6, article 13, 2014.

[18] M. Kuhn, D. Szklarczyk, S. Pletscher-Frankild et al., "STITCH 4: integration of protein-chemical interactions with user data," Nucleic Acids Research, vol. 42, no. 1, pp. D401-D407, 2014.

[19] C. Qin, C. Zhang, F. Zhu et al., "Therapeutic target database update 2014: a resource for targeted therapeutics," Nucleic Acids Research, vol. 42, no. 1, pp. D1118-D1123, 2014.

[20] D. Szklarczyk, A. Franceschini, S. Wyder et al., "STRING v10: protein-protein interaction networks, integrated over the tree of life," Nucleic Acids Research, vol. 43, no. 1, pp. D447-D452, 2015.

[21] L. Salwinski, L. Licata, A. Winter et al., "Recurated protein interaction datasets," Nature Methods, vol. 6, no. 12, pp. 860861, 2009.

[22] S. Orchard, M. Ammari, B. Aranda et al., "The MIntAct project-IntAct as a common curation platform for 11 molecular interaction databases," Nucleic Acids Research, vol. 42, no. 1, pp. D358-D363, 2014.

[23] L. Licata, L. Briganti, D. Peluso et al., "MINT, the molecular interaction database: 2012 update," Nucleic Acids Research, vol. 40, no. 1, pp. D857-D861, 2012.

[24] F. Zhao, L. Guochun, Y. Yang, L. Shi, L. Xu, and L. Yin, "A network pharmacology approach to determine active ingredients and rationality of herb combinations of Modified-Simiaowan for treatment of gout," Journal of Ethnopharmacology, vol. 168, pp. 1-16, 2015.

[25] M. Franz, C. T. Lopes, G. Huck, Y. Dong, O. Sumer, and G. D. Bader, "Cytoscape.js: a graph theory library for visualisation and analysis," Bioinformatics, vol. 32, no. 2, pp. 309-311, 2016.

[26] P. V. Missiuro, K. Liu, L. Zou et al., "Information flow analysis of interactome networks," PLoS Computational Biology, vol. 5, no. 4, 2009.

[27] K. Raman, N. Damaraju, and G. K. Joshi, “The organisational structure of protein networks: Revisiting the centrality-lethality hypothesis," Systems and Synthetic Biology, vol. 8, no. 1, pp. 7381, 2014.

[28] Y. Zhang, M. Bai, B. Zhang et al., "Uncovering pharmacological mechanisms of Wu-tou decoction acting on rheumatoid arthritis through systems approaches: drug-target prediction, network analysis and experimental validation," Scientific Reports, vol. 5, article 9463, 2015.

[29] D. W. Huang, B. T. Sherman, and R. A. Lempicki, "Systematic and integrative analysis of large gene lists using DAVID bioinformatics resources," Nature Protocols, vol. 4, no. 1, pp. 44-57, 2009.

[30] M. B. Goldring and M. Otero, "Inflammation in osteoarthritis," Current Opinion in Rheumatology, vol. 23, no. 5, pp. 471-478, 2011.
[31] C. J. Vangsness, W. S. Burke, S. J. Narvy, R. D. MacPhee, and A. N. Fedenko, "Human knee synovial fluid cytokines correlated with grade of knee osteoarthritis-a pilot study," Bulletin of the NYU Hospital for Joint Diseases, vol. 69, no. 2, pp. 122-127, 2011.

[32] M. B. Mueller and R. S. Tuan, "Anabolic/Catabolic balance in pathogenesis of osteoarthritis: identifying molecular targets," $P M$ \& $R$, vol. 3, no. 6, supplement 1, pp. S3-S11, 2011.

[33] C. A. Dinarello, "Immunological and inflammatory functions of the interleukin-1 family," Annual Review of Immunology, vol. 27, pp. 519-550, 2009.

[34] K. S. Santangelo, G. J. Nuovo, and A. L. Bertone, "In vivo reduction or blockade of interleukin- $1 \beta$ in primary osteoarthritis influences expression of mediators implicated in pathogenesis," Osteoarthritis and Cartilage, vol. 20, no. 12, pp. 1610-1618, 2012.

[35] M. Suzuki, M. Hashizume, H. Yoshida, M. Shiina, and M. Mihara, "IL-6 and IL-1 synergistically enhanced the production of MMPs from synovial cells by up-regulating IL- 6 production and IL-1 receptor I expression," Cytokine, vol. 51, no. 2, pp. 178183,2010

[36] P. K. Rai, A. K. Singh, O. P. Singh, N. P. Rai, and A. Dwivedi, "Efficacy of leech therapy in the management of osteoarthritis (Sandhivata)," Ayu, vol. 32, no. 2, pp. 213-317, 2011.

[37] I. García-Arnandis, M. I. Guillén, F. Gomar, J.-P. Pelletier, J. Martel-Pelletier, and M. J. Alcaraz, "High mobility group box 1 potentiates the pro-inflammatory effects of interleukin- $1 \beta$ in osteoarthritic synoviocytes," Arthritis Research \& Therapy, vol. 12, no. 4, article R165, 2010.

[38] G. I. Wassilew, U. Lehnigk, G. N. Duda, W. R. Taylor, G. Matziolis, and C. Dynybil, "The expression of proinflammatory cytokines and matrix metalloproteinases in the synovial membranes of patients with osteoarthritis compared with traumatic knee disorders," Arthroscopy, vol. 26, no. 8, pp. 1096-1104, 2010.

[39] M. Kapoor, J. Martel-Pelletier, D. Lajeunesse, J.-P. Pelletier, and H. Fahmi, "Role of proinflammatory cytokines in the pathophysiology of osteoarthritis," Nature Reviews Rheumatology, vol. 7, no. 1, pp. 33-42, 2011.

[40] M. Attur, S. Krasnokutsky-Samuels, J. Samuels, and S. B. Abramson, "Prognostic biomarkers in osteoarthritis," Current Opinion in Rheumatology, vol. 25, no. 1, pp. 136-144, 2013.

[41] C. A. Dinarello, A. Simon, and J. W. M. van der Meer, “Treating inflammation by blocking interleukin-1 in a broad spectrum of diseases," Nature Reviews Drug Discovery, vol. 11, no. 8, pp. 633652, 2012.

[42] R. Tao, S. Wang, X. Xia et al., "Pyrroloquinoline quinone slows down the progression of osteoarthritis by inhibiting nitric oxide production and metalloproteinase synthesis," Inflammation, vol. 38, no. 4, pp. 1546-1555, 2015.

[43] M. B. Goldring, "The role of the chondrocyte in osteoarthritis," Arthritis \& Rheumatism, vol. 43, no. 9, pp. 1916-1926, 2000.

[44] Y. Kobayashi, "The regulatory role of nitric oxide in proinflammatory cytokine expression during the induction and resolution of inflammation," Journal of Leukocyte Biology, vol. 88, no. 6, pp. 1157-1162, 2010.

[45] M. Tomita, E. F. Sato, M. Nishikawa, Y. Yamano, and M. Inoue, "Nitric oxide regulates mitochondrial respiration and functions of articular chondrocytes," Arthritis and Rheumatism, vol. 44, no. 1, pp. 96-104, 2001.

[46] M. A. Cake, R. C. Appleyard, R. A. Read, P. Ghosh, M. V. Swain, and G. C. Murrell, "Topical administration of the nitric oxide donor glyceryl trinitrate modifies the structural and biomechanical properties of ovine articular cartilage," Osteoarthritis and Cartilage, vol. 11, no. 12, pp. 872-878, 2003. 
[47] M. R. Sowers, D. McConnell, M. Jannausch, A. G. Buyuktur, M. Hochberg, and D. A. Jamadar, "Estradiol and its metabolites and their association with knee osteoarthritis," Arthritis and Rheumatism, vol. 54, no. 8, pp. 2481-2487, 2006.

[48] V. K. Srikanth, J. L. Fryer, G. Zhai, T. M. Winzenberg, D. Hosmer, and G. Jones, "A meta-analysis of sex differences prevalence, incidence and severity of osteoarthritis," Osteoarthritis and Cartilage, vol. 13, no. 9, pp. 769-781, 2005.

[49] X. Dai, C. Wang, J. Dai et al., "Association of single nucleotide polymorphisms in estrogen receptor alpha gene with susceptibility to knee osteoarthritis: a case-control study in a Chinese Han population," BioMed Research International, vol. 2014, Article ID 151457, 12 pages, 2014.

[50] J. A. Roman-Blas, S. Castañeda, R. Largo, and G. HerreroBeaumont, "Osteoarthritis associated with estrogen deficiency," Arthritis Research \& Therapy, vol. 11, no. 5, article 241, 2009.

[51] M. Martín-Millán and S. Castañeda, "Estrogens, osteoarthritis and inflammation," Joint Bone Spine, vol. 80, no. 4, pp. 368-373, 2013.

[52] Y. H. Sniekers, H. Weinans, G. J. V. M. van Osch, and J. P. T. M. van Leeuwen, "Oestrogen is important for maintenance of cartilage and subchondral bone in a murine model of knee osteoarthritis," Arthritis Research \& Therapy, vol. 12, no. 5, article R182, 2010.

[53] M. G. Sørensen, K. Henriksen, M. H. Dziegiel, L. B. Tankó, and M. A. Karsdal, "Estrogen directly attenuates human osteoclastogenesis, but has no effect on resorption by mature osteoclasts," DNA and Cell Biology, vol. 25, no. 8, pp. 475-483, 2006.

[54] K. D. Ham, R. F. Loeser, B. R. Lindgren, and C. S. Carlson, "Effects of long-term estrogen replacement therapy on osteoarthritis severity in cynomolgus monkeys," Arthritis \& Rheumatism, vol. 46, no. 7, pp. 1956-1964, 2002.

[55] D. A. Pampena, K. A. Robertson, O. Litvinova, G. Lajoie, H. A. Goldberg, and G. K. Hunter, "Inhibition of hydroxyapatite formation by osteopontin phosphopeptides," Biochemical Journal, vol. 378, no. 3, pp. 1083-1087, 2004.

[56] E. Calvo, S. Castañeda, R. Largo, M. E. Fernández-Valle, F. Rodríguez-Salvanés, and G. Herrero-Beaumont, "Osteoporosis increases the severity of cartilage damage in an experimental model of osteoarthritis in rabbits," Osteoarthritis and Cartilage, vol. 15, no. 1, pp. 69-77, 2007.

[57] K. D. Ham, R. F. Loeser, B. R. Lindgren, and C. S. Carlson, "Effects of long-term estrogen replacement therapy on osteoarthritis severity in cynomolgus monkeys," Arthritis and Rheumatism, vol. 46, no. 7, pp. 1956-1964, 2002.

[58] F. E. Watt, "Hand osteoarthritis, menopause and menopausal hormone therapy," Maturitas, vol. 83, pp. 13-18, 2016.

[59] M. A. Karsdal, A. C. Bay-Jensen, K. Henriksen, and C. Christiansen, "The pathogenesis of osteoarthritis involves bone, cartilage and synovial inflammation: may estrogen be a magic bullet?" Menopause International, vol. 18, no. 4, pp. 139-146, 2012.

[60] C. Ma, Y. Zhang, Y.-Q. Li, C. Chen, W. Cai, and Y.-L. Zeng, “The role of PPAR $\gamma$ in advanced glycation end products-induced inflammatory response in human chondrocytes," PLOS ONE, vol. 10, no. 5, Article ID e0125776, 2015.

[61] A. Haseeb and T. M. Haqqi, "Immunopathogenesis of osteoarthritis," Clinical Immunology, vol. 146, no. 3, pp. 185-196, 2013.

[62] T. Kato, S. Miyaki, H. Ishitobi et al., "Exosomes from IL-1 $\beta$ stimulated synovial fibroblasts induce osteoarthritic changes in articular chondrocytes," Arthritis Research \& Therapy, vol. 16, no. 4, article R163, 2014.

[63] T. Piao, Z. Ma, X. Li, and J. Liu, "Taraxasterol inhibits IL$1 \beta$-induced inflammatory response in human osteoarthritic chondrocytes," European Journal of Pharmacology, vol. 756, pp. 38-42, 2015.

[64] B. Yang, X. Kang, Y. Xing et al., "Effect of microRNA-145 on ILlbeta-induced cartilage degradation in human chondrocytes," FEBS Letters, vol. 588, no. 14, pp. 2344-2352, 2014.

[65] X. Zhang, X. Xu, T. Xu, and S. Qin, “ $\beta$-ecdysterone suppresses interleukin-1 $\beta$-induced apoptosis and inflammation in rat chondrocytes via inhibition of NF- $\kappa \mathrm{B}$ signaling pathway," Drug Development Research, vol. 75, no. 3, pp. 195-201, 2014.

[66] L. Assis, L. P. Milares, T. Almeida et al., "Aerobic exercise training and low-level laser therapy modulate inflammatory response and degenerative process in an experimental model of knee osteoarthritis in rats," Osteoarthritis and Cartilage, vol. 24, no. 1, pp. 169-177, 2016.

[67] F. Guilak, B. Fermor, F. J. Keefe et al., "The role of biomechanics and inflammation in cartilage injury and repair," Clinical Orthopaedics and Related Research, no. 423, pp. 17-26, 2004.

[68] S. B. Abramson, "Nitric oxide in inflammation and pain associated with osteoarthritis," Arthritis Research \& Therapy, vol. 10, supplement 2, article S2, 2008.

[69] V. Balaganur, N. N. Pathak, M. C. Lingaraju et al., "Effect of Smethylisothiourea, an inducible nitric oxide synthase inhibitor, in joint pain and pathology in surgically induced model of osteoarthritis," Connective Tissue Research, vol. 55, no. 5-6, pp. 367-377, 2014.

[70] C. M. Hancock and C. Riegger-Krugh, "Modulation of pain in osteoarthritis: the role of nitric oxide," Clinical Journal of Pain, vol. 24, no. 4, pp. 353-365, 2008.

[71] V. Balaganur, N. N. Pathak, M. C. Lingaraju et al., "Chondroprotective and anti-inflammatory effects of S-methylisothiourea, an inducible nitric oxide synthase inhibitor in cartilage and synovial explants model of osteoarthritis," Journal of Pharmacy and Pharmacology, vol. 66, no. 7, pp. 1021-1031, 2014.

[72] L. Xu, C. Sun, S. Zhang et al., "Sam68 promotes NF- $\kappa$ B activation and apoptosis signaling in articular chondrocytes during osteoarthritis," Inflammation Research, vol. 64, no. 11, pp. 895-902, 2015.

[73] T. Aigner, S. Söder, P. M. Gebhard, A. McAlinden, and J. Haag, "Mechanisms of Disease: role of chondrocytes in the pathogenesis of osteoarthritis-structure, chaos and senescence," Nature Clinical Practice Rheumatology, vol. 3, no. 7, pp. 391-399, 2007.

[74] R. F. Loeser, "Aging and osteoarthritis: the role of chondrocyte senescence and aging changes in the cartilage matrix," Osteoarthritis and Cartilage, vol. 17, no. 8, pp. 971-979, 2009.

[75] Y. Zhou, S.-Q. Liu, L. Yu et al., "Berberine prevents nitric oxideinduced rat chondrocyte apoptosis and cartilage degeneration in a rat osteoarthritis model via AMPK and p38 MAPK signaling," Apoptosis, vol. 20, no. 9, pp. 1187-1199, 2015.

[76] Y. Li, F. Zhang, C. Zeng et al., "Autophagy in osteoarthritis," Joint Bone Spine, vol. 83, no. 2, pp. 143-148, 2016.

[77] H. Zhao, T. Zhang, C. Xia et al., "Berberine ameliorates cartilage degeneration in interleukin-1 $\beta$-stimulated rat chondrocytes and in a rat model of osteoarthritis via Akt signalling," Journal of Cellular and Molecular Medicine, vol. 18, no. 2, pp. 283-292, 2014. 


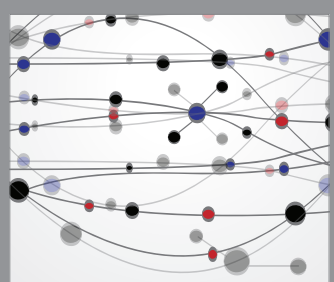

The Scientific World Journal
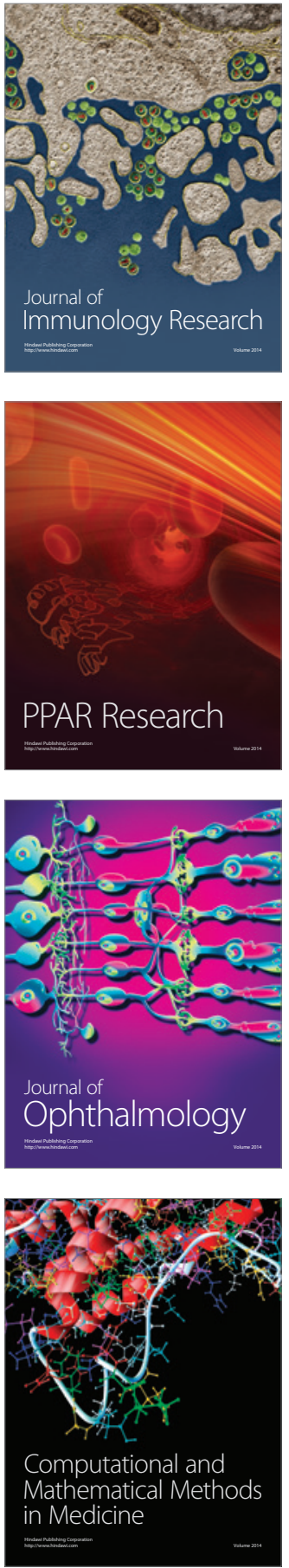

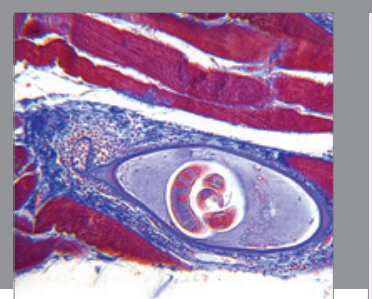

Gastroenterology Research and Practice

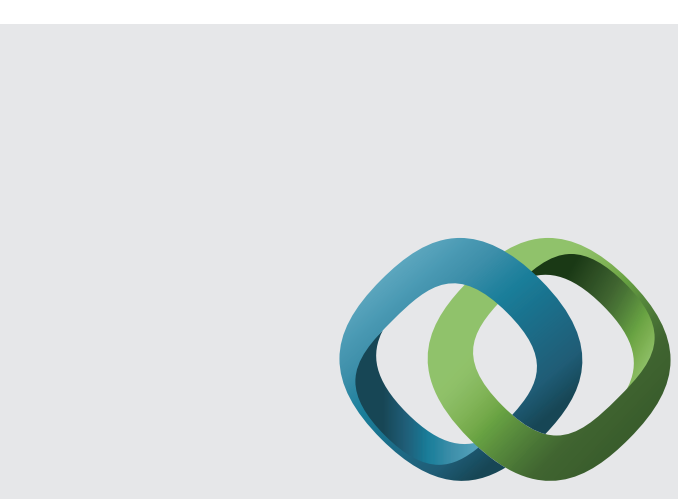

\section{Hindawi}

Submit your manuscripts at

http://www.hindawi.com
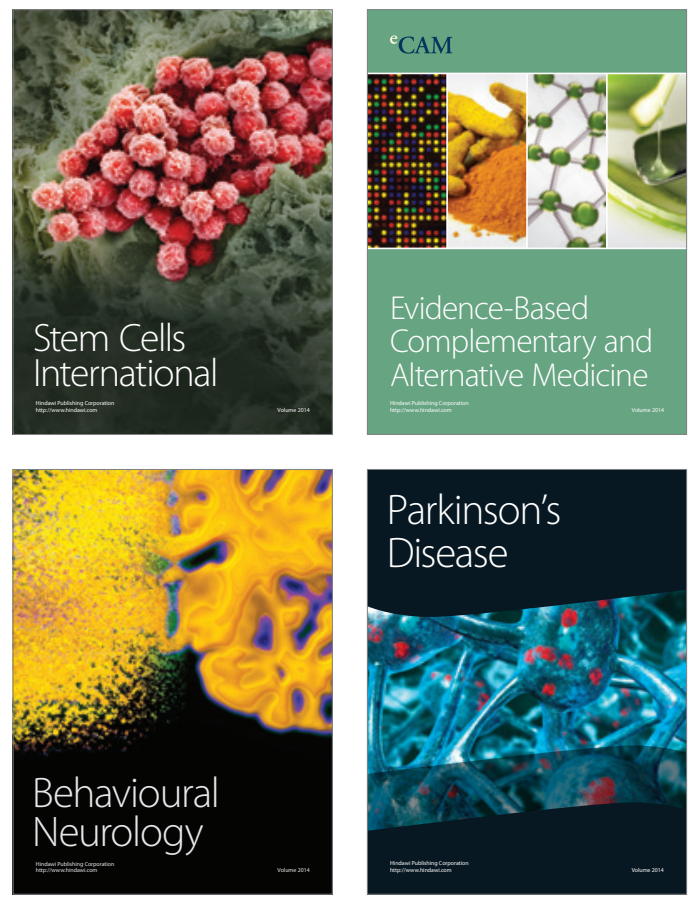
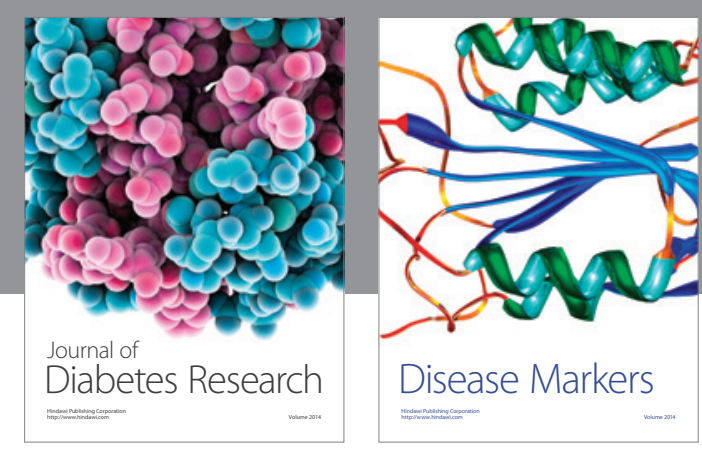

Disease Markers
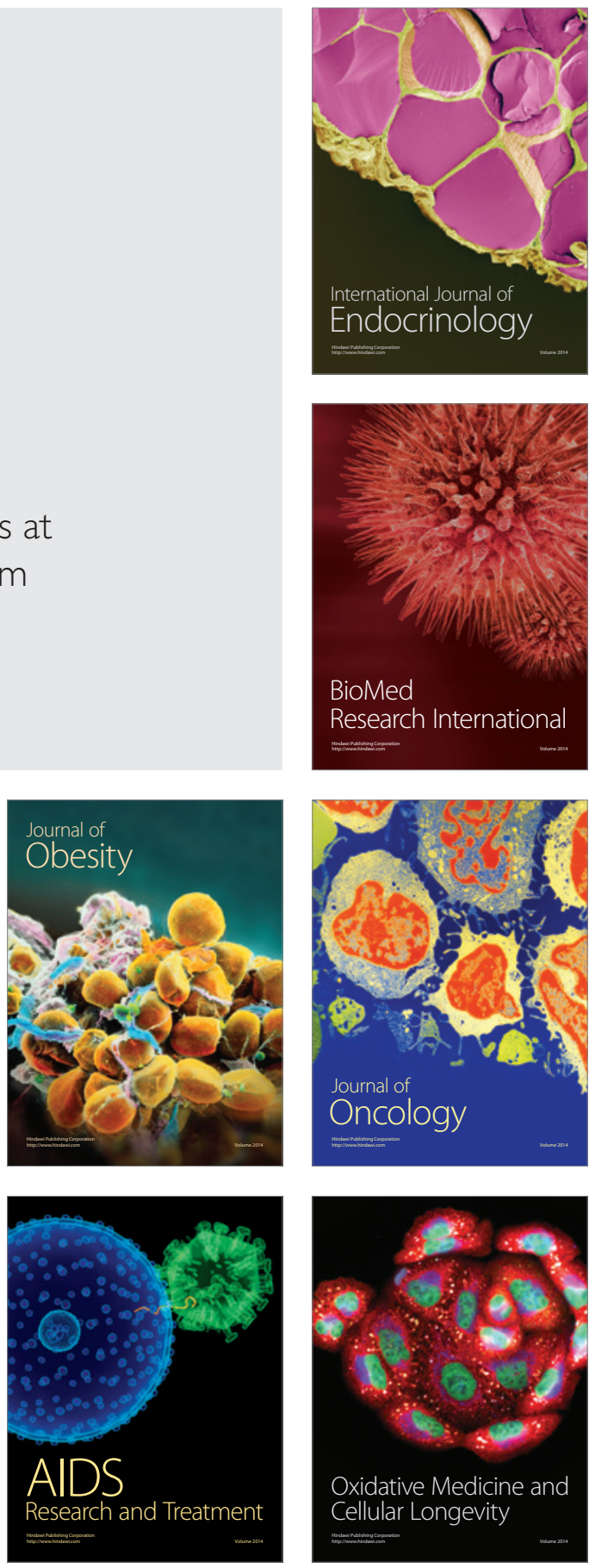